\title{
Use of Natural Products in the Prevention and Treatment of Denture Stomatitis
}

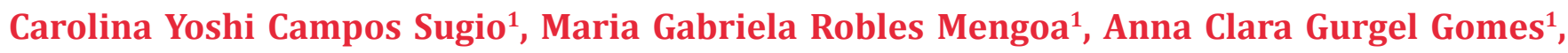 \\ Amanda Aparecida Maia Neves Garcia ${ }^{1}$, Thaís Marchini de Oliveira ${ }^{2}$ and Karin Hermana \\ Neppelenbroek ${ }^{1 *}$ \\ ${ }^{1}$ Department of Prosthodontics and Periodontics, Bauru School of Dentistry, University of São Paulo, Brazil \\ ${ }^{2}$ Department of Pediatric Dentistry, Orthodontics and Public Health, Bauru School of Dentistry, University of São Paulo, Brazil
}

\begin{abstract}
Despite the wide variety of antifungal agents for the denture stomatitis (DS) treatment, they may induce hepatotoxic and nephrotoxic effects, allergic reactions and interactions with other medicaments, besides the emergence of antifungal resistance to the most commonly used classes of drugs. For these reasons, natural products present as an interesting therapeutic alternative. This narrative review summarizes the use of main natural products in the prevention and treatment of DS, which are obtained from propolis, Pelargonium graveolens, Equisetum giganteum, Punica granatum, and Melaleuca alternifolia. These substances are of ancient use in the medical field and, recently, have been widely reported in different dental specialties, mainly for their anti-inflammatory and antifungal action. Despite the favourable results obtained in the literature with such substances, the scientific evidence for the use of natural products in Dentistry, especially for the purpose of DS therapy is not conclusive, requiring further clinical studies to consolidate their application.
\end{abstract}

KEYWORDS: Candida; Denture stomatitis; Medicinal plants; Natural products

\section{INTRODUCTION}

The most common clinical manifestation of oral candidiasis is denture stomatitis (DS), a lesion characterized by inflammation and erythema in the oral mucosa under the denture base [1]. This condition has a prevalence of up to $70 \%$ in patients with removable acrylic dentures [1,2], and its aetiology is multifactorial, involving general and local factors, however, it is strongly related to colonization by Candida spp. [3]. Although Candida spp. are physiologically present in the bacterial flora of the oral cavity, in case of an imbalance between the host and fungus, the normal conditions of the mucosa may change to a parasitic situation, resulting, in oral lesions such as DS. Predisposing factors for this change include systemic disease, immune deficiencies, reduced salivary flow, use of broad-spectrum antibiotics, continued use of denture at night, especially old and poorly adapted prostheses, smoking, poor oral and dental hygiene [3-5]. DS treatment requires multiple approaches with topical antifungal therapy, oral care, hygiene procedures and denture disinfection, adjustment of anatomical irregularities and/or denture base, making of new denture and discontinuation of nocturnal denture use
Quick Response Code:

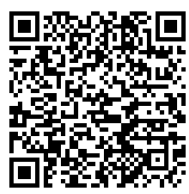

Address for correspondence: Karin Hermana Neppelenbroek, Department of Prosthodontics and Periodontics, University of São Paulo, Brazil

Received: January 24, 2020 Published: February 26, 2020

How to cite this article: Carolina Yoshi Campos Sugio1, Maria Gabriela Robles Mengoa1, Anna Clara Gurgel G, Amanda Aparecida Maia Neves G, Thaís Marchini de O, Karin Hermana N. Use of Natural Products in the Prevention and Treatment of Denture Stomatitis. 2020 - 2(1) OAJBS.ID.000146. DOI: 10.38125/OAJBS.000146 
$[4,6]$. Within antifungal therapy, various agents are used such as nystatin, amphotericin B, clotrimazole, miconazole, itraconazole, fluconazole, and ketoconazole $[7,8]$. However, in the conventional form of topical and systemic treatment, a high recurrence rate has been reported $[9,10]$, due to the difficulty in maintaining therapeutic concentration [10], the strict dosage, especially for elderly patients [11] and the maintenance of contact between the contaminated acrylic base [10]. Furthermore, a genetic adaptation of fungi to these drugs has been reported, developing antimicrobial resistance [12].

Due to such disadvantages, alternative therapies have been proposed, including the addition of antifungal agents to temporary soft materials. This protocol has shown promising results for DS treatment because it has prolonged the clinical longevity of the liners, prevented the accumulation of denture biofilm $[13,14]$ and inhibited Candida albicans colonization by breaking the cycle of mucosal reinfection through denture. Despite these advantages, consideration should also be given to the possibility of these drugs inducing hepatotoxic and nephrotoxic effects [15], as well as allergic reactions and interactions with other medications. Although such effects are minimized or eliminated by direct drug release at the site of infection [8], as when incorporating them into soft materials, the use of natural-based medicines as an alternative to antifungals / antimicrobials for DS treatment has been recently recommended [16].

A wide variety of plant extracts have shown antifungal activity against Candida, suggesting its potential for DS treatment $[16,17]$ Some natural products such as Propolis used in gel form, mouthwash or mucoadhesive [18], Punica granatum gel [19], Pelargonium graveolens olive oil [20] and Ricinus communis in mouthwash form [21] have shown the same efficacy as conventional antifungals in treating patients with DS. Thus, it would be pertinent to review the use of main natural products in the prevention and treatment of DS.

\section{USE OF NATURAL PRODUCTS AND THEIR BENEFITS}

Studies involving the use of natural products for the treatment against Candida began to be more relevant from 2006, highlighting the interest of reaching treatment alternatives to allopathic drugs in cases of fungal infections. Considering their effective antimicrobial and antifungal activity and their anti-inflammatory and antioxidant effects, these substances may be an option for synthetic chemicals with less harmful potential and fewer adverse effects on humans [16]. Among natural products, plants contain components with biologically active molecules, which result in essential oil extracts with potential antifungal activity [22-24]. Moreover, compounds such as terpenes have shown to hinder biofilm formation, while monoterpenes such as carvacrol, geraniol, and thymol associated with other components are effective in the treatment of candidiasis. Terpenoids have excellent activity against hyphae forms of $C$. albicans at concentrations not toxic to HeLa cells whereas components obtained from plants such as saponins, peptides, alkaloids, and proteins have antifungal activity [25].

Thus, the use of these substances in Dentistry has been studied. In endodontics, natural products have been investigated for cleaning and disinfecting root canals, intracanal medication, cementing agent, removal of filling material and pulp and dentin repair, among others. Polyphenolics showed potential anticariogenic properties without interrupt the main health benefits of the resident oral microbiota [26]. They constitute one of the most common and widespread groups found in all vegetative organs, as well as in flowers and fruits. Polyphenolics have shown to be able to eliminate bacteria or inhibit biofilm, while others natural substances revealed the ability to regulate demineralization or remineralization of dental hard tissue [26]. However, it has been demonstrated that natural products are less effective at preventing caries than traditional chemical agents such as antimicrobials or fluorine. Further studies on the mechanism of action of the effective anti-cariogenic compounds of most natural products with these properties have not been additionally developed [26].

Due to their antibiotic and anti-inflammatory properties, the use of medicinal plants in the treatment of periodontal diseases has been suggested. In Ashouri et al. [27] clinical trial, the results indicated that local application of Aloe vera gel can be considered as an adjunctive treatment of chronic periodontitis. In addition, some in situ studies have shown that commercial green tea and a rinse green tea extract, polyphenol-rich products have been able to reduce erosive and erosive/abrasive wear. Their use and other synthetic inhibitors have been suggested to control dentin matrix loss [28].

\section{MAJOR NATURALPRODUCTS INTHE PREVENTION AND TREATMENT OF DENTURE STOMATITIS}

For many years a wide variety of natural products have traditionally been used as antifungal agents, especially those of the genus Candida [16,17,29]. Considering that oral candidiasis is a frequent opportunistic infection mainly caused by Candida colonization [1], the use of these substances may play an important role in the DS treatment. The following natural products have shown potential for the prevention and treatment of DS.

\section{Propolis}

Propolis is a complex mixture of resinous and balsamic material, usually collected by bees from flowers and tree exudate, which, in the hive, are mixed with salivary secretions. Besides the antimicrobial effect of this compound [16], antibacterial, antifungal, anti-inflammatory, antioxidant and immunoregulatory properties are known. In the Dentistry field, studies have evaluated the biological and curative activities of propolis [16], its role in inhibiting biofilm formation and preventing dental caries [30], its use as an intra-canal drug in endodontic treatment, among others [16]. These properties also drew attention to the possible DS treatment.

In the study by Santos et al. [29], the propolis gel formula showed efficacy compared to Daktarin, suggesting to be an alternative topical DS treatment. Similarly, the randomized clinical study by Pina et al. [18] recommended the formulation of propolis as an alternative in the treatment of elderly patients with DS. The meta-analysis performed by Hwu et al. [31] gathered available scientific evidence on the effects of propolis use on oral health, including oral infection, plaque, and DS. It was compared the use of gels, mouthwashes or propolis-based tablets with placebos, other mouthwashes or gels and daily hygiene care. The results showed a reduction in oral infection with a reduction in Candida and Streptococcus mutans quantification, as well as supragingival plaque index and clinical signs of DS. The authors observed the effectiveness of propolis mouthwash, being comparable to other mouthwashes, such as chlorhexidine and nystatin.

\section{Pelargonium Graveolens Olive 0il}

Pelargonium or geranium olive oil belongs to the Geraniaceae family [32] and it is characterized by being non-toxic, non-irritating, 
non-sensitizing, with no adverse effects when administered [32]. Its main constituents are citronellol and geraniol, which are responsible for their biological activities [32] and anti-inflammatory and antifungal effects, especially against C. albicans [20]. Pelargonium extracts have antibacterial and antioxidant action that justify their use in food and cosmetic industries [20]. A randomized clinical trial proposed a treatment using topical gel of $P$. graveolens in patients users of complete denture with DS. The authors suggested that the topical formulation had some beneficial therapeutic effects in DS treatment [20].

\section{Equisetum Giganteum}

E. giganteum popularly known as 'horsetail' is an endemic plant from Central and South America. Some studies have shown their antimicrobial effects against Streptococcus pyogenes, Bacullis cereus, Bacillus subtilis, Enterococcus faecalis, Staphylococcus aureus, and Staphylococcus epidermidis [33]. When added to denture fixative powder, E. giganteum hydroethanolic extracts influenced the development of $C$. albicans biofilm on the thermopolymerizable acrylic resin surface, significantly minimizing its colonization and reducing its metabolism [34]. In the in vitro study by Alavarce et al. [17], E. giganteum extract showed non-stick activity in C. albicans biofilms in experimental models simulating complete dentures. In addition, all tested concentrations of the extract showed antiinflammatory activity and no cytotoxicity was observed in contact with human cells (monocytes). Another similar recent study by da Silva et al. [33] evaluated the in vitro non-stick activity of $E$. giganteum extract on C. albicans biofilm. Results revealed a 15-44\% reduction in biofilm mass and decrease in CFU/mL (79\%) compared to untreated control. An important non-stick activity was observed in C. albicans biofilm at all concentrations. The results showed that the plant can be considered a potential alternative for topical and preventive treatment against oral candidiasis $[17,33]$.

\section{Punica Granatum}

P. granatum Linné, also known as pomegranate, is a fruit deciduous shrub or small Asian tree of the Punicaceae family. Rich in bioactive compounds, they can be used to treat cancer, cardiovascular disease, oral disease, bacterial infections, diabetes mellitus, diarrhea, UV radiation skin diseases and haemorrhoids $[35,36]$. Still, the plant has several phytotherapeutic compounds such as flavonoids, polyphenols, organic acids, and tannins, mainly in its bark [36,37]. Due to its antimicrobial, antifungal, antiviral, antioxidant, anti-inflammatory, anti-carcinogenic and other therapeutic properties, $P$. granatum has gained attention for the treatment of oral cavity diseases [37]. Due to its antifungal action, it has been shown that $P$. granatum bark extract could be recommended in DS treatment [38]. Madugula et al. [37] evaluated the antifungal efficacy in vitro of P. granatum bark extract compared to cotrimazole at its minimum inhibitory concentrations (MICs). The MIC of P. granatum bark extract was as efficient as that of cotrimazole and, therefore, recommended for candidiasis therapy. In a clinical study, de Souza Vasconcelos et al. [19] concluded that $P$ granatum extract can be recommended as an antifungal agent in DS treatment.

\section{Melaleuca Alternifolia}

M. alternifolia, also known as tea tree oil, is a multipurpose medicinal herb whose essential oil can be obtained from its leaves by steam distillation. Such a compound has shown promising effect as a topical antifungal agent in the treatment of dandruff, acne and oral candidosis [39]. Tobouti et al. [40] conducted a study on the antimicrobial properties and activity against $M$. alternifolia and Copaíba oil candidiasis and concluded that both oils have activity against $C$. albicans, inhibiting the adhesion of their biofilm, in prevention against DS. Moreover, the combination of tissue conditioners with $M$. alternifolia essential oil showed an efficient fungicidal action against $C$. albicans according to the in vivo study by Catalán et al. [41]. In this research, 27 patients with DS had their prostheses relined with Coe-Comfort which was used without association or modified by M. alternifolia or nystatin. After 12 days, the results showed that the group whose complete dentures were relined with the material modified by the natural product showed total inhibition of $C$. albicans, in addition to a significant reduction in palate inflammation compared to the conventional antifungal group. It was concluded that M. alternifolia oil combined with CoeComfort tissue conditioner was effective in DS treatment.

\section{Ricinus Communis}

R. communis is a plant that presents tissue biocompatibility, as well as bactericidal and antifungal properties. Popularly known as 'castor bean', this plant is characteristic of tropical climate areas and from its seeds, it is possible to obtain an antimicrobial and anti-inflammatory olive oil [42]. In traditional medicine, it has been used in cases of abdominal disorders, arthritis, rheumatism, insomnia, constipation, the expulsion of the placenta and back pain, muscle pain, chronic lumbar and sciatica, chronic head, gallbladder, and menstrual period [42]. Dental studies have used castor oil as a root canal irrigating solution, toothbrush for complete dentures, mouthwash in DS treatment and as a sanitizer $[12,43,44]$. In one of these studies, the effectiveness of the plant with nystatin and miconazole was compared in the treatment of elderly patients diagnosed with DS [12]. It was concluded that $R$. communis may improve the clinical status of DS in elderly patients with results comparable to those of miconazole [12]. In another randomized clinical trial, Arruda et al. [45] evaluated 50 patients with complete dentures and diagnosed with DS. Participants were instructed to brush their dentures (brush and soap) and soak them in $0.85 \%$ saline, $0.1 \%$ sodium hypochlorite, $0.2 \%$ sodium hypochlorite, or $8 \%$ R. communis. All tested solutions were effective in reducing signs of DS, but both sodium hypochlorite solutions were the most effective solutions for the biofilm control. In the clinical investigation of Badaró et al. [46], the effectiveness of $R$. communis was compared to sodium hypochlorite solution in relation to anti-biofilm action, antimicrobial activity, patient satisfaction and remission of clinical signs of DS. It was shown that the solution obtained from the plant in a concentration of $10 \%$ and that of $0.25 \%$ sodium hypochlorite were effective in removing the biofilm, improvement of the DS clinical signs and reducing the fungal load, being equally accepted by the patient.

\section{DISCUSSION}

In the oral cavity, Candida microorganisms are part of the normal flora, however, when there are predisposing factors that generate imbalance in the normality of the local environment, this relationship can create a parasitism situation and generate diseases such as DS in patients who use complete dentures [3-5]. This pathology is considered the most found of oral candidiasis [1] and is also considered the most frequent oral mucosal lesion in the elderly [2]. A variety of methods and therapies have been proposed for DS treatment, being the most recommended the use of topical and systemic antifungals and oral hygiene, cleaning and disinfection instructions $[5,6,15]$. The greatest challenge in the management of this clinical condition is related to the high 
rates of clinical relapse and post-treatment recurrence, especially when antifungal agents are used [9]. Thus, the treatment should be individualized to the patient, considering both the associated predisposing factors and the need to combine more than one therapeutic approach. Although systemic antifungal therapy is recommended for immunocompromised patients, adverse health implications should be considered, as they may result in hepatotoxic and nephrotoxic effects, as well as drug interactions [15]. Recurrence of Candida spp. infection in the oral mucosa and denture bases has been widely reported in the short and longterm following discontinuation of systemic therapies [47]. The failure occurs mainly because the drugs do not reach an enough concentration for fungal elimination on the denture surfaces, which leads to substrate recolonization and, consequently, of the supporting mucosa. Similarly, topical antifungal therapy with agents such as miconazole and especially, nystatin $[4,15]$ also has limitations, although it is still the most common treatment used for DS, as discussed in this review article. Thus, the incorporation of these drugs into temporary soft denture materials has been suggested with potential therapy for DS, as antimicrobial/antifungal agents are released gradually from the modified polymeric matrix to the infected sites, remaining in effective concentration for a period similar to conventional treatment with topical medication $[11,13,14]$. Despite the advantages presented with this protocol for DS treatment, even at lower concentrations and directed to the site of infection, it is possible that side effects may arise. Considering the possible toxicity, drug-drug interactions, and appearance of drug-resistant fungal species, as previously mentioned $[9,12]$, investigations into antimicrobial products have intensified in recent decades. Considering the previously mentioned aspects, this literature review aimed to discuss natural products with antifungal effects for Candida species aiming at their therapeutic potential for DS. From the literature consulted, it was possible to highlight some main extracts of plants and natural substances with action against Candida and potential for DS treatment $[16,17,33]$. The antimicrobial and curative action of propolis has been proven in various fields of Dentistry and, specifically referring to DS, clinical studies have demonstrated [18,29] its effectiveness, based on clinical and microbiological results obtained, which were comparable to a conventional allopathic antifungal agent (miconazole). Despite these favourable outcomes, the authors of the only systematic review with meta-analysis on propolis use in oral health available in the literature concluded that further studies are needed to state whether the product can replace synthetic substances in the treatment of oral infection, dental plaque and DS [31]. In a study of elderly patients with DS, it was observed that $R$. communis oil in mouthwash improved clinical status but was not effective in significantly reducing fungal load during evaluation [21]. Similarly, this substance also improved clinical signs of the pathology but was less effective than $0.1 \%$ sodium hypochlorite in controlling denture biofilm [45]. However, when the mouthwash was prepared at a higher concentration (10\%), it was as effective as $0.25 \%$ sodium hypochlorite solution for biofilm removal, remission of clinical signs of DS and reduction of fungal load [46]. Although attesting the great therapeutic potential of $R$. communis oil for DS, the contradictory findings of previous research still suggest new clinical studies for the indication of the product as an effective method for the prevention and treatment of the lesion. P. graveolens oil has a synergistic in vitro action comparable to ketoconazole [20] and presents inhibitory effect on Candida colonization [20]. A randomized clinical trial in DS patients demonstrated a partial or total improvement of the condition for most of them according to microbiological and clinical outcomes after 14 days of using a 1\% P. graveolens topical gel formulation. Despite these beneficial therapeutic effects, the authors recommended the development of further studies to increase the patient sample, especially for the analysis of possible adverse effects of the product.

In addition to producing C. albicans antibiofilm activity in vitro [40], M. alternifolia oil associated with Coe-Comfort tissue conditioner produced comparable microbiological and clinical results to nystatin in combination with the same material in one study in patients with DS [41]. In an in vitro research, P. granatum bark extract was as effective as clotrimazole in inhibiting Candida from saliva samples from patients with oral candidiasis [37]. The gel extract of P. granatum showed performance similar to miconazole for the inhibition of Candida spp. in patients with DS, having been recommended as a therapeutic alternative for this condition [19]. Therefore, it is noted that although both natural products performed similarly to synthetic antifungal drugs, only one clinical study of each was found in the relevant literature, thus lacking information for a definitive indication for DS treatment.

In combination with a denture powder adhesive, E. giganteum and P. granatum hydroethanolic extract reduced the colonization of C. albicans on the thermopolymerized acrylic resin, amplifying the anti-biofilm action of the adhesive on the specimens by up to 12 h [34]. E. giganteum hydroethanolic showed non-stick properties [17] and reduced biomass and viable cells in C. albicans biofilms [33] on denture base resin specimens. These findings suggest the potential of this plant for DS treatment, but clinical studies are needed to confirm this hypothesis.

Despite the known antifungal properties of natural products highlighted in this literature review, there are still studies in vitro and especially in vivo on their effect against Candida colonization and biofilm, as well as the prevention and treatment of DS so this alternative therapy can be recommended instead of the conventional one.

\section{CONCLUSION}

According to the literature reviewed, it was concluded that propolis, P. graveolens, E. giganteum, P. granatum, M. alternifolia and $R$. communis can be highlighted among natural products with potential for prevention and, especially, for DS treatment due to their in vitro inhibitory actions on the colonization and development of Candida biofilm, as well the clinical reduction of the fungal load and the improvement of the clinical signs of DS. Despite these favourable results, there is still little scientific evidence, especially based on clinical studies, for natural product therapy to be recommended as a substitute for the conventional treatment of DS with synthetic antifungal agents.

\section{ACKNOWLEDGEMENT}

The present study was supported by São Paulo Research Foundation-FAPESP (grants 2017/07314-1 and 2017/22996-1).

\section{REFERENCES}

1. Gendreau L, Loewy ZG (2011) Epidemiology and etiology of denture stomatitis. J Prosthodont 20(4): 251-260.

2. Rivera C, Droguett D, Arenas-Márquez MJ (2017) Oral mucosal lesions in a Chilean elderly population: A retrospective study with a systematic review from thirteen countries. J Clin Exp Dent 9(2): e276-e283.

3. Martori E, Ayuso-Montero R, Martinez-Gomis J, Viñas M, Peraire M (2014) Risk factors for denture-related oral mucosal lesions in a geriatric population. J Prosthet Dent 111(4): 273-279. 
4. Neppelenbroek KH, Pavarina AC, Palomari Spolidorio DM, Sgavioli Massucato EM, Spolidorio LC, et al. (2008) Effectiveness of microwave disinfection of complete dentures on the treatment of Candida-related denture stomatitis. J Oral Rehabil 35(11): 836-846.

5. Ferreira MA, Pereira-Cenci T, Rodrigues de Vasconcelos LM, RodriguesGarcia RC, Del Bel Cury AA (2009) Efficacy of denture cleansers on denture liners contaminated with Candida species. Clin Oral Investig 13(2): 237-242.

6. Srinivasan M, Gulabani M (2010) A microbiological evaluation of the use of denture cleansers in combination with an oral rinse in complete denture patients. Indian J Dent Res 21(3): 353-356.

7. Bueno MG, Urban VM, Barbério GS, da Silva WJ, Porto VC, et al. (2015) Effect of antimicrobial agents incorporated into resilient denture relines on the Candida albicans biofilm. Oral Dis 21(1): 57-65.

8. Hotta J, Garlet GP, Cestari TM, Lima JFM, Porto VC, et al. (2019) In vivo biocompatibility of an interim denture resilient liner containing antifungal drugs. J Prosthet Dent 121(1): 135-142.

9. Ramage G, Tomsett K, Wickes BL, López-Ribot JL, Redding SW (2004) Denture stomatitis: a role for Candida biofilms. Oral Surg Oral Med Oral Pathol Oral Radiol Endod 98(1): 53-59.

10. Yarborough A, Cooper L, Duqum I, Mendonça G, McGraw K, et al. (2016) Evidence regarding the treatment of denture stomatitis. J Prosthodont 25(4): 288-301.

11. Truhlar MR, Shay K, Sohnle P (1994) Use of a new assay technique for quantification of antifungal activity of nystatin incorporated in denture liners. J Prosthet Dent 71(5): 517-524.

12. Goldman G, da Silva Ferreira M, dos Reis Marques E, Savoldi M, Perlin D, et al (2004) Evaluation of fluconazole resistance mechanisms in Candida albicans clinical isolates from HIV-infected patients in Brazil. Diagn Microbiol Infect Dis 50(1): 25-32.

13. Schneid TR (1992) An in vitro analysis of a sustained release system for the treatment of denture stomatitis. Spec Care Dentist 12(6): 245-250.

14. Nikawa H, Hamada T, Yamamoto T, Kumagai H (1997) Effects of salivary or serum pellicles on the Candida albicans growth and biofilm formation on soft lining materials in vitro. J Oral Rehabil 24(8): 594-604.

15. Lombardi T, Budtz-Jorgensen E (1993) Treatment of denture-induced stomatitis: a review. Eur J Prosthodont Restor Dent 2(1): 17-22.

16. Casaroto AR, Lara VS (2010) Phytomedicines for Candida-associated denture stomatitis. Fitoterapia 81(5): 323-328.

17. Alavarce RA, Saldanha LL, Almeida NL, Porto VC, Dokkedal AL, et al. (2015) The beneficial effect of Equisetum giganteum L. against Candida biofilm formation: new approaches to denture stomatitis. Evid Based Complement Alternat Med 2015: 939625.

18. Pina GM, Lia EN, Berretta AA, Nascimento AP, Torres EC, et al. (2017) Efficacy of propolis on the denture stomatitis treatment in older adults: a multicentric randomized trial. Evid Based Complement Alternat Med 2017: 8971746.

19. Vasconcelos LC, Sampaio MC, Sampaio FC, Higino JS (2003) Use of Punica granatum as an antifungal agent against candidosis associated with denture stomatitis. Mycoses 46(5-6): 192-196.

20. Sabzghabaee AM, Shirdare Z, Ebadian B, Aslani A, Ghannadi A (2011) Clinical evaluation of the essential oil of Pelargonium graveolens for the treatment of denture stomatitis. Dent Res J (Isfahan) 8(Suppl 1): S1055108.

21. Pinelli LA, Montandon AA, Corbi SC, Moraes TA, Fais LM (2013) Ricinus communis treatment of denture stomatitis in institutionalised elderly. J Oral Rehabil 40(5): 375-380.

22. Zida A, Bamba S, Yacouba A, Ouedraogo-Traore R, Guiguemdé RT (2017) Anti-Candida albicans natural products, sources of new antifungal drugs: A review. J Mycol Med 27(1): 1-19.

23. Dalleau S, Cateau E, Bergès T, Berjeaud JM, Imbert C (2008) In vitro activity of terpenes against Candida biofilms. Int J Antimicrob Agents 31(6): 572-576.
24. Manohar V, Ingram C, Gray J, Talpur NA, Echard BW, et al. (2001) Antifungal activities of origanum oil against Candida albicans. Mol Cell Biochem 228(1-2): 111-117.

25. Groppo FC, Bergamaschi CC, Cogo K, Franz-Montan M, Motta RH, et al. (2008) Use of phytotherapy in dentistry. Phytother Res 22(8): 993-998.

26. Cheng L, Li J, He L, Zhou X (2015) Natural products and caries prevention. Caries Res 49(Suppl 1): 38-45.

27. Ashouri MA, Radafshar G, Jahandideh Y, Kakaei N (2017) Clinical evaluation of effects of local application of aloe vera gel as an adjunct to Scaling and root planning in patients with chronic periodontitis. J Dent (Shiraz) 18(3): 165-172.

28. Kato MT, Magalhães AC, Rios D, Hannas AR, Attin T, et al. (2009) Protective effect of green tea on dentin erosion and abrasion. J Appl Oral Sci. 17(6): 560-564.

29. Santos VR, Gomes RT, De Mesquita RA, De Moura MDG, França EC, et al. (2008) Efficacy of Brazilian propolis gel for the management of denture stomatitis: a pilot study. Phytother Res 22(11): 1544-1547.

30. Duarte S, Rosalen PL, Hayacibara MF, Cury JA, Bowen WH, et al. (2006) The influence of a novel propolis on mutans streptococci biofilms and caries development in rats. Arch Oral Biol 51(1): 15-22.

31. Hwu YJ, Lin FY (2014) Effectiveness of propolis on oral health: a metaanalysis. J Nurs Res 22(4): 221-229.

32. Giongo JL, de Almeida Vaucher R, Fausto VP, Quatrin PM, Lopes LQS, et al. (2016) Anti-Candida activity assessment of Pelargonium graveolens oil free and nano-emulsion in biofilm formation in hospital medical supplies. Microb Pathog 100: 170-178.

33. Da Silva RA, Bernardo LP, Moreno JML, Lara VS, Porto VC (2017) Equisetum giganteum influences the ability of Candida albicans in forming biofilms over the denture acrylic resin surface. Pharm Biol 55(1): 1698-1702.

34. Almeida NLM, Saldanha LL, da Silva RA, Pinke KH, da Costa EF, et al. (2018) Antimicrobial activity of denture adhesive associated with Equisetum giganteum and Punica granatum enriched fractions against Candida albicans biofilms on acrylic resin surfaces. Biofouling 34(1): 6273.

35. Kaur G, Jabbar Z, Athar M, Alam MS (2006) Punica granatum (pomegranate) flower extract possesses potent antioxidant activity and abrogates Fe-NTA induced hepatotoxicity in mice. Food Chem Toxicol 44(7): 984-993.

36. Jurenka JS (2008) Therapeutic applications of pomegranate (Punica granatum L.): a review. Altern Med Rev 13(2): 128-144.

37. Madugula P, Reddy S, Koneru J, Rao AS, Sruthi R, et al. (2017) "Rhetoric to Reality"- Efficacy of Punica Granatum peel extract on oral candidiasis: an in vitro study. J Clin Diagn Res 11(1): ZC114-ZC117.

38. Bassiri-Jahromi S, Katiraee F, Hajimahmoodi M, Mostafavi E, Talebi M, et al. (2015) In vitro antifungal activity of various persian cultivars of Punica granatum L. Extracts against Candida species. Jundishapur J Nat Pharm Prod 10: e19754.

39. Vankadara SK, Hallikerimath RB, Patil V, Bhat K, Doddamani MH (2017) Effect of Melaleuca alternifolia mixed with tissue conditioners in varying doses on colonization and Inhibition of Candida albicans: an in vitro study. Contemp Clin Dent 8(3): 446-450.

40. Tobouti PL, Mussi MCM, Rossi DCP, Pigatti FM, Taborda CP, et al. (2016) Influence of melaleuca and copaiba oils on Candida albicans adhesion. Gerodontology 33(3): 380-385.

41. Catalán A, Pacheco JG, Martínez A, Mondaca MA (2008) In vitro and In vivo activity of melaleuca alternifolia mixed with tissue conditioner on Candida albicans. Oral Surg Oral Med Oral Pathol Oral Radiol Endod 105(3): 327-332

42. Marwat SK, Rehman F, Khan EA, Baloch MS, Sadiq M, et al. (2017) Review-Ricinus cmmunis - Ethnomedicinal uses and pharmacological activities. Pak J Pharm Sci 30(5): 1815-1827.

43. Meneghin MP, Nomelini SM, Sousa-Neto MD, Marchesan MA, França SC, 
et al. (2006) Morphologic and morphometric analysis of the root canal apical third cleaning after biomechanical preparation using 3.3\% Ricinus communis detergent and $1 \% \mathrm{NaOCl}$ as irrigating solutions. J Appl Oral Sci 14(3): 178-182.

44. Andrade IM, Andrade KM, Pisani MX, Silva-Lovato CH, de Souza RF, et al. (2014) Trial of an experimental castor oil solution for cleaning dentures. Braz Dent J 25(1): 43-47.

45. Arruda CNF, Salles MM, Badaró MM, de Cássia OV, Macedo AP, et al. (2017) Effect of sodium hypochlorite and Ricinus communis solutions on control of denture biofilm: A randomized crossover clinical trial. J Prosthet Dent 117(6): 729-734.

46. Badaró MM, Salles MM, Leite VMF, Arruda CNF, Oliveira VC, et al. (2017) Clinical trial for evaluation of Ricinus communis and sodium hypochlorite as denture cleanser. J Appl Oral Sci 25: 324-334.

47. Arikan A, Kulak Y, Kadir T (1995) Comparison of different treatment methods for localized and generalized simple denture stomatitis. J Oral Rehabil 22(5): 365-369. 